\title{
Vacuum energy and repulsive Casimir forces in quantum star graphs
}

\author{
S. A. Fulling, ${ }^{1, *}$ L. Kaplan, ${ }^{2, \dagger}$ and J. H. Wilson ${ }^{1, \dagger}$ \\ ${ }^{1}$ Departments of Mathematics and Physics, Texas A\&M University, College Station, Texas 77843-3368, USA \\ ${ }^{2}$ Department of Physics, Tulane University, New Orleans, Louisiana 70118, USA
}

(Received 15 August 2006; revised manuscript received 26 March 2007; published 24 July 2007)

\begin{abstract}
Casimir pistons are models in which finite Casimir forces can be calculated without any suspect renormalizations. It has been suggested that such forces are always attractive, but we present several counterexamples, notably a simple type of quantum graph in which the sign of the force depends upon the number of edges. We also show that Casimir forces in quantum graphs can be reliably computed by summing over the classical orbits, and study the rate of convergence of the periodic orbit expansion. In generic situations where no analytic expression is available, the sign and approximate magnitude of Casimir forces can often be obtained using only the shortest classical orbits.
\end{abstract}

DOI: 10.1103/PhysRevA.76.012118

PACS number(s): 03.65.Sq, 03.70.+k, 11.10.Kk, 42.25.Gy

\section{INTRODUCTION}

According to a classic calculation [1], the Casimir force inside a roughly cubical rectangular parallelepiped is repulsive; that is, it tends to expand the box. The reasoning leading to this conclusion is open to criticism on two related grounds: It ignores the possibility of nontrivial vacuum energy in the region outside the box, and it involves "renormalization" in the sense of discarding divergent terms associated with the boundary although (unlike the case of parallel plates, or any calculation of forces between rigid bodies) the geometry of the boundary depends upon the dimensions of the box. Recently (see also Ref. [2]) a class of scenarios called "Casimir pistons" has been introduced to which these objections do not apply. The piston is an idealized plate that is free to move along a rectangular shaft, whose length $L$ $-a$ to the right of the piston is taken arbitrarily large (Fig. 1). Both the external region and the divergent (or cutoffdependent) terms in the internal vacuum energy are independent of the piston position $a$ so that a well defined, finite force on the piston is calculated. One finds that this force is always attractive, both for a two-dimensional scalar-field model with the Dirichlet boundary condition [3] and for a three-dimensional electromagnetic field with the perfectconductor boundary condition [4].

Barton [5] showed that the piston force can be repulsive for some (not too small) values of $a$ if the conducting material is replaced by a weakly polarizable dielectric. This result is somewhat ironic in that one reason for suspicion of repulsive Casimir forces is the belief that the force between disjoint bodies of realistically modeled material should always be attractive. The unexpected result is easily understood, however, as being due to attraction between the piston and the distant part of the shaft. The effect would disappear if the shaft extended a long distance to the left of the fixed plate ("baffle") at $a=0$ as well as to the right of the piston.

\footnotetext{
*URL: http://www.math.tamu.edu/ fulling; fulling@math.tamu.edu

†URL: http://www.tulane.edu/ lkaplan;

lkaplan@tulane.edu

¥jwilson.thequark@gmail.com
}

In the present paper we study the vacuum energy and Casimir forces in one-dimensional quantum graph models and observe several situations with idealized boundary conditions for which the piston force is unambiguously repulsive. In quantum graphs of high symmetry, the Casimir forces may be calculated analytically. More generally, we show that these forces may be obtained systematically from a sum over the classical periodic orbits in the graph, as done in three-dimensional problems in Refs. [6-8], and we discuss the rate of convergence of the periodic-orbit expansion. In some cases, the sign and approximate magnitude of the force on a Casimir piston may be obtained using only the shortest orbit hitting that piston. Although the quantum graph models are less realistic than those studied in Refs. [4,5], they do show that repulsive Casimir forces do arise physically and are not inevitably an artifact of a naive renormalization scheme. Our effects are unrelated to that in Ref. [5] and do not depend on the asymmetry noted above in connection with that paper. The periodic-orbit techniques discussed here have relevance to the study of Casimir energies in more realistic geometries (see Ref. [9]), including two- and threedimensional chaotic billiards. In an appendix, we consider a situation in which an unambiguously repulsive Casimir force appears for the electromagnetic field in a three-dimensional geometry. Throughout, we take $\hbar=1=c$.

\section{VACUUM ENERGY IN QUANTUM GRAPHS}

A finite quantum graph [10-13] consists of $B$ onedimensional undirected bonds or edges of length $L_{j}(j$ $=1, \ldots, B)$. Either end of each bond ends at one of $V$ vertices, and the valence $v_{\alpha} \geqslant 1$ of a vertex is defined as the

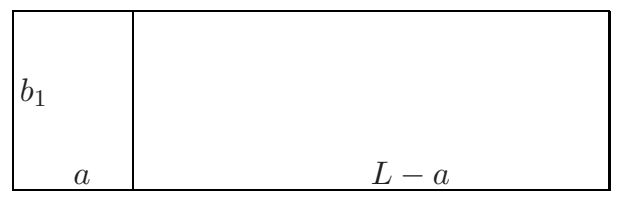

FIG. 1. A rectangular piston in two dimensions (see Ref. [3]). In three dimensions there is another length $b_{2}$ perpendicular to the plane of the figure. 
number of bonds meeting there. A normal mode $u$ of the quantum graph has the form $u_{j}(x)=a_{j} \cos \left(k x_{j}\right)+b_{j} \sin \left(k x_{j}\right)$ on every bond $j$, and satisfies the specified boundary conditions at each vertex. Despite their simplicity, quantum graph models have previously shed light on a number of important physical problems, having served originally as models of conjugated molecules, and more recently of quantum, electromagnetic, and acoustic waveguides and circuits. These models have also served as valuable testing grounds for studying more general properties of quantum behavior, including Anderson localization, quantum chaos, adiabatic quantum transport, and scattering. A recent review may be found in Ref. [14].

In the spirit of abstract modeling, the vacuum energy of a graph is defined as the sum (renormalized) of zero-point energies over all normal-mode frequencies $\omega_{n}$, where the frequency $\omega_{n}$ is equal to the wave number $k_{n}$ in our units. It is convenient to apply an exponential ultraviolet regularization (the same answer would be obtained, for example, by a calculation with zeta functions):

$$
E(t) \equiv \sum_{n=0}^{\infty} \frac{1}{2} \omega_{n} e^{-\omega_{n} t}=-\frac{1}{2} \frac{d}{d t} T(t),
$$

where

$$
T(t) \equiv \sum_{n=0}^{\infty} e^{-\omega_{n} t}
$$

is the trace of the so-called cylinder kernel [15].

\section{ANALYTIC EXAMPLES OF REPULSIVE CASIMIR FORCES}

\section{A. One-dimensional piston with mixed boundary conditions}

The first example is already rather well known, in its essence. Consider a scalar field quantized on a line divided into three parts by two points, at each of which either a Dirichlet or a Neumann boundary condition is imposed. The contributions of the two infinite (or, better, extremely long) intervals to the Casimir force will vanish. (As emphasized in Ref. [4], the force contributed by a long shaft is entirely associated with periodic orbits perpendicular to the shaft, which do not exist in the one-dimensional case.) Let the length of the central interval be $a$. Then the frequencies of the normal modes are

$$
\omega_{n}=\frac{n \pi}{a}
$$

for nonnegative (or positive) integer $n$, if the boundaries are both Neumann (or both Dirichlet, respectively), and one has

$T(t)=\sum_{n=0,1}^{\infty} e^{-\pi n t / a}=\frac{1}{1-e^{-\pi t / a}}[-1]=\frac{a}{\pi t} \pm \frac{1}{2}+\frac{1}{12} \frac{\pi t}{a}+O\left(t^{2}\right)$.

Thus the regularized vacuum energy is

$$
E(t)=\frac{a}{2 \pi t^{2}}-\frac{\pi}{24 a}+O(t) .
$$

The leading, divergent term is proportional to the interval length $a$ and corresponds to a geometry-independent constant energy density. This term is compensated in the force by similar terms in the exterior regions, already discarded. Then letting $t \rightarrow 0$, we obtain the well-known attractive force

$$
F \equiv-\frac{\partial E}{\partial a}=-\frac{\pi}{24 a^{2}}
$$

More precisely, if the entire space has length $L$, then the regularized energy of the exterior regions is

$$
\frac{L-a}{2 \pi t^{2}}+O\left(L^{-1}\right)
$$

The second term is negligible as $L \rightarrow \infty$, and the first term combines with the first term of Eq. (5) to make a term independent of $a$, which, therefore, is an unobservable constant energy shift that contributes nothing to the force. Henceforth we shall not repeat this type of argument every time it is needed, and will simply refer to such endpoints as Neumann or Dirichlet pistons.

On the other hand, if one boundary is Dirichlet and the other Neumann, then the eigenfrequencies are

$$
\omega_{n}=\frac{(2 n+1) \pi}{2 a} .
$$

The same calculation leads to

$$
T(t)=e^{-\pi t / 2 a} \sum_{n=0}^{\infty} e^{-\pi n t / a}=\frac{1}{2 \sinh (\pi t / 2 a)}=\frac{a}{\pi t}-\frac{1}{24} \frac{\pi t}{a}+O\left(t^{2}\right) ;
$$

the regularized energy is

$$
E(t)=\frac{a}{2 \pi t^{2}}+\frac{\pi}{48 a}+O(t),
$$

and the force comes out to be repulsive:

$$
F=+\frac{\pi}{48 a^{2}} \text {. }
$$

\section{B. Quantum star graphs}

In the next model the space consists of $B$ one-dimensional rays of large length $L$ attached to a central vertex (Fig. 2). In each ray a Neumann piston is located a distance $a$ from the vertex, so that a normal mode of the field in ray $j$ must take the form $u_{j}(x)=c_{j} \cos [\omega(x-a)]$ when $x$ is measured from the center. At the central vertex the field has the Kirchhoff (generalized Neumann) behavior

$$
u_{j}(0)=C \text { for all } j, \quad \sum_{j=1}^{B} u_{j}^{\prime}(0)=0 .
$$

The following analysis is part of a broader study of vacuum energy in quantum graphs [16] (see also Refs. [17-19]). 


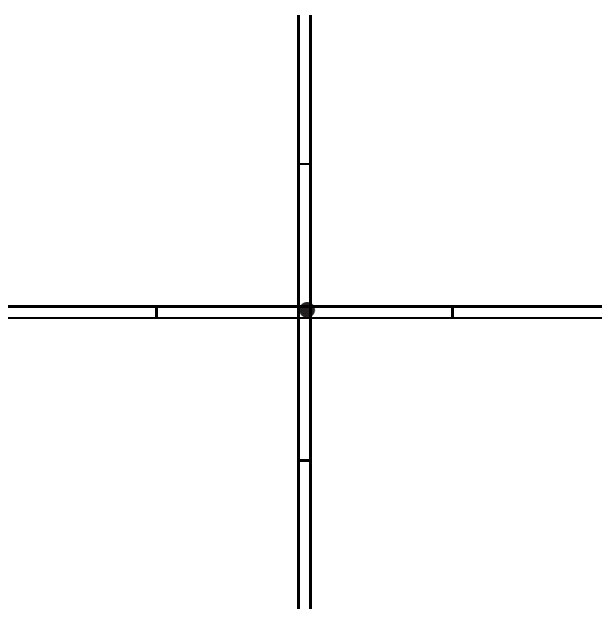

FIG. 2. A star graph with a piston installed in each edge. (The pistons are actually points; the edges have no thickness.)

There are two types of normal modes. First, if $\cos (\omega a)$ $\neq 0$, we have from Eq. (12) that $c_{j}=C / \cos (\omega a)$ and $\tan (\omega a)=0$, whence $\omega$ is one of the numbers (3). Second, if $\cos (\omega a)=0$, then $\omega$ is one of the numbers (8) and

$$
\sum_{j=1}^{B} c_{j}=0
$$

which has $B-1$ independent solutions. Therefore, the energies and forces are just the appropriate linear combinations of those calculated in the previous example: the regularized energy for the whole system is

$$
E(t)=\frac{B L}{2 \pi t^{2}}+\frac{(B-3) \pi}{48 a}+O\left(L^{-1}\right)+O(t)
$$

and the force [either from Eq. (14) or from Eqs. (6) and (11)] is

$$
F=-\frac{\pi}{24 a^{2}}+(B-1) \frac{\pi}{48 a^{2}}=\frac{(B-3) \pi}{48 a^{2}} .
$$

When $B=1$ or 2 , the result reduces properly to that for an ordinary Neumann interval of length $a$ or $2 a$, respectively. When $B>3$, however, the force is repulsive: if the pistons are free to all move together, they will tend to move outward. (More generally, a periodic-orbit calculation, such as discussed in Sec. IV, is applicable to unequal piston displacements and confirms that the force on each individual piston is outward, so there are no other, asymmetrical modes that are partly attractive.) This repulsive effect cannot be attributed to mixed boundary conditions, since all the conditions are of the Neumann type. [However, replacing all the pistons with Dirichlet pistons while maintaining Eq. (12) would interchange the roles of the two types of eigenvalues and produce attraction for all $B>1$.]

\section{PERIODIC-ORBIT CALCULATIONS FOR GENERAL GRAPHS}

For a general quantum graph, e.g., for a star graph with unequal bond lengths or with more complicated boundary conditions, no simple expressions for the normal-mode frequencies $\omega_{n}$ are available, and thus the vacuum energy and Casimir forces cannot be computed in closed form. Computing the spectrum numerically, as discussed below, allows for an accurate evaluation of the vacuum energy for any specific quantum graph, but this type of brute force calculation must be repeated anew for every geometry and does not provide much physical insight regarding the attractive or repulsive character of Casimir forces in different cases. Instead, much intuition may be obtained using a classical-orbit approach, where the sign and magnitude of every contribution to the vacuum energy are seen to be directly related to bond lengths and boundary conditions at the vertices.

It is convenient to describe boundary conditions at every vertex $\alpha$ by a unitary $v_{\alpha} \times v_{\alpha}$ scattering matrix $\sigma_{\alpha}$ (which acts on the space of undirected bonds meeting at vertex $\alpha$ ). For example, a Neumann or Dirichlet boundary condition at a vertex of valence $v_{\alpha}=1$ corresponds to a scattering matrix $\sigma_{\alpha}=(+1)$ or $(-1)$, respectively, while the Kirchhoff boundary condition is described by $\left(\sigma_{\alpha}\right)_{j j^{\prime}}=\frac{2}{v_{\alpha}}-\delta_{j j^{\prime}}$. Together these constitute a $2 B \times 2 B$ scattering matrix $S$ for the entire graph of $2 B$ directed bonds [11,13] or bond ends [20-22]. To make the following arguments valid, we must assume that $S$ is independent of energy or frequency ( $k$-independent), as is true for the Dirichlet, Neumann, and Kirchhoff boundary conditions we treat here (but not for the more general Kirchhoff-type boundary conditions where a potential is attached to each vertex $[11,12,17,23])$. Then one can construct $[11,13]$ a trace formula relating the spectrum of a graph (away from the point $\omega=0$, which makes no contribution to vacuum energy anyway) to its periodic orbits

$$
\sum_{n} \delta\left(\omega-\omega_{n}\right)=\frac{L}{\pi}+\operatorname{Re} \frac{1}{\pi} \sum_{p} \sum_{r=1}^{\infty}\left(A_{p}\right)^{r} L_{p} e^{i r \omega L_{p}} .
$$

(Variations on the trace formula have been found in Refs. $[10,22,16]$, and elsewhere.) In Eq. (16) the values $\omega_{n}$ are the normal-mode frequencies, $L=\sum_{j=1}^{B} L_{j}$ is the total length of the graph, which determines the smooth (Weyl) contribution to the spectrum, and the sum over $p$ is a sum over primitive periodic orbits (orbits that cannot be written as repetitions of shorter orbits). Each $p$ takes the form $p=j_{1} j_{2} \cdots j_{n}$, where every $j_{i}$ is a directed bond. The corresponding amplitude of the primitive periodic orbit is given by a product of scattering factors $A_{p}=S_{j_{1} j_{2}} \cdots S_{j_{n-1} j_{n}} S_{j_{n} j_{1}}$, the metric length of the primitive orbit is $L_{p}=L_{j_{1}}+\cdots+L_{j_{n}}$, and each $r$ is a different repetition number of our base primitive orbit.

Substituting the spectrum given by Eq. (16) into Eq. (1), we obtain

$$
E(t)=\frac{L}{2 \pi t^{2}}-\operatorname{Re} \frac{1}{2 \pi} \sum_{p} \sum_{r=1}^{\infty} \frac{\left(A_{p}\right)^{r}}{L_{p} r^{2}}+O(t) .
$$

As discussed previously, the finite vacuum energy, which is relevant for computation of Casimir forces, is obtained by 
dropping the divergent Weyl term and taking the limit $t \rightarrow 0$,

$$
E_{c}=-\frac{1}{2 \pi} \operatorname{Re} \sum_{p} \sum_{r=1}^{\infty} \frac{\left(A_{p}\right)^{r}}{L_{p} r^{2}} .
$$

A mathematically rigorous derivation and proof of (conditional) convergence of Eq. (18) will appear in Ref. [19].

Equivalently, we may begin with the free cylinder kernel in one dimension

$$
T_{0}\left(x, x^{\prime}, t\right)=\frac{t}{\pi} \frac{1}{\left(x-x^{\prime}\right)^{2}+t^{2}},
$$

apply the method of images to include scattering from the vertices, take the trace

$$
T(t)=\int d x T(x, x, t)=\frac{t}{\pi} \frac{L}{t^{2}}+\operatorname{Re} \sum_{p} \sum_{r=1}^{\infty} \frac{t}{\pi} \frac{2 L_{p}\left(A_{p}\right)^{r}}{\left(r L_{p}\right)^{2}}+O\left(t^{2}\right),
$$

and finally use Eq. (1) to obtain the result (17). (This construction, which generalizes the study of the heat kernel in Ref. [10], is described in detail in Ref. [16].)

The Casimir force on any piston may be obtained easily by differentiating Eq. (18) term by term with respect to the appropriate bond length $L_{j}$.

We note that the expansion (18) of the vacuum energy is exact and involves periodic orbits only. The derivation of Eq. (20) hinges on the identity $\left(\sigma_{\alpha}\right)^{2}=I$ for the scattering matrix at each vertex. This condition holds for any $k$-independent scattering matrix [20,21], including real scattering matrices of the form used here, but also complex energy-independent scattering matrices in the case of time-reversal symmetry breaking by magnetic fields. It is the crucial ingredient in proving that closed but nonperiodic paths (i.e., paths that start and end at $x$ but with opposite momenta) make no net contribution to the vacuum energy. When $S$ depends on $k$, two complications arise. First, the method of images cannot be so easily applied to "time-domain" integral kernels such as $T$ and the heat kernel, because the reflection law becomes nonlocal in $t$. Second, the identity $\left(\sigma_{\alpha}\right)^{2}=I$ no longer applies, and the nonperiodic paths make a nontrivial contribution to the vacuum energy [and to the density of states, (16), even when $\omega \neq 0]$. Both effects are visible in the investigations of the simplest special cases in Refs. [17,24].

To evaluate the accuracy of the periodic-orbit expansion in situations where no analytic expression for the vacuum energy is available, we may compare with a brute-force calculation where the spectrum is evaluated numerically. For a general $V$-vertex graph, the normal-mode frequencies are given by solutions of a characteristic equation det $h(\omega)=0$, where $h(\omega)$ is a $V \times V$ matrix [11]. For the special case of a star graph with irrationally related bond lengths, we have

$$
\sum_{j=1}^{B} \tan \left(\omega L_{j}+\theta_{j}\right)=0
$$

where $\theta_{j}=0$ or $\pi$ for a Neumann or Dirichlet piston on bond $j$, respectively. In any case, given a method for obtaining a numerical spectrum $\omega_{n}$, we may evaluate

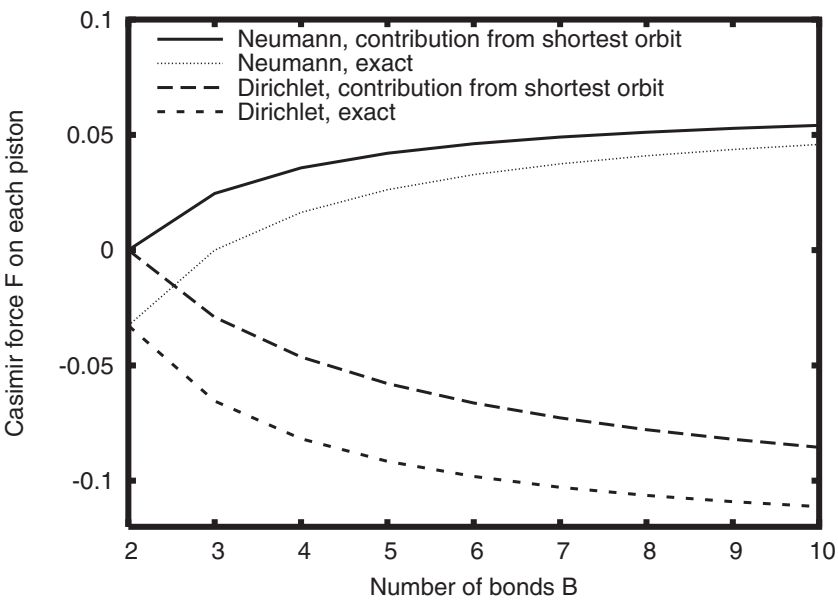

FIG. 3. The force on a piston in a star graph with $B$ bonds of length 1, Kirchhoff boundary condition at the center, and either Neumann or Dirichlet boundary condition at each piston is computed using only the shortest periodic orbit [Eq. (24)] and compared with the exact answer. Positive values indicate repulsive forces. All quantities plotted in this and subsequent figures are dimensionless.

$$
E_{\text {finite }}(t)=\sum_{n} \frac{1}{2} \omega_{n} e^{-\omega_{n} t}-\frac{L}{2 \pi t^{2}}
$$

to any desired accuracy by summing over all $\omega_{n} \leqslant \omega_{\max }$ where $\omega_{\max } \gg 1 / t$. Since the divergent term associated with the Weyl density of states, or equivalently with the free onedimensional geometry, has already been subtracted, we only need take the numerical limit $t \rightarrow 0$ to obtain the true vacuum energy $E_{c}$. Expressing the regularized vacuum energy as a power series

$$
E_{\text {finite }}(t)=E_{c}+\alpha_{1} t+\alpha_{2} t^{2}+\cdots,
$$

we may apply Richardson extrapolation to approximate the vacuum energy to any desired order of accuracy $E_{c}$ $=E_{c}^{\text {numerical }}+O\left(t^{s}\right)$, by evaluating $E_{\text {finite }}(t)$ at $s$ distinct values of the regularization parameter $t$.

\section{RATE OF CONVERGENCE OF PERIODIC-ORBIT EXPANSION}

We consider a star graph with Kirchhoff boundary condition for $B$ bonds meeting at the central vertex, and a Dirichlet or Neumann piston on each bond at a distance $a_{j}$ from the central vertex (i.e., the pistons may be located at different distances from the center). The leading contribution to the vacuum energy is given by the shortest primitive orbits, each of which travels back and forth along a single bond. Including all repetitions of such orbits, we obtain

$$
E_{c}^{\text {shortest }}=-\frac{1}{4 \pi} \sum_{j=1}^{B} \sum_{r=1}^{\infty} \frac{1}{r^{2}}\left(\frac{2}{B}-1\right)^{r} \frac{\cos \left(r \theta_{j}\right)}{a_{j}},
$$

where $\theta_{j}=0$ for a Neumann piston or $\pi$ for a Dirichlet piston. For example, for all Neumann pistons the sum over $r$ can be evaluated as a dilogarithm, which in turn can be expanded in powers of $1 / B$ as 


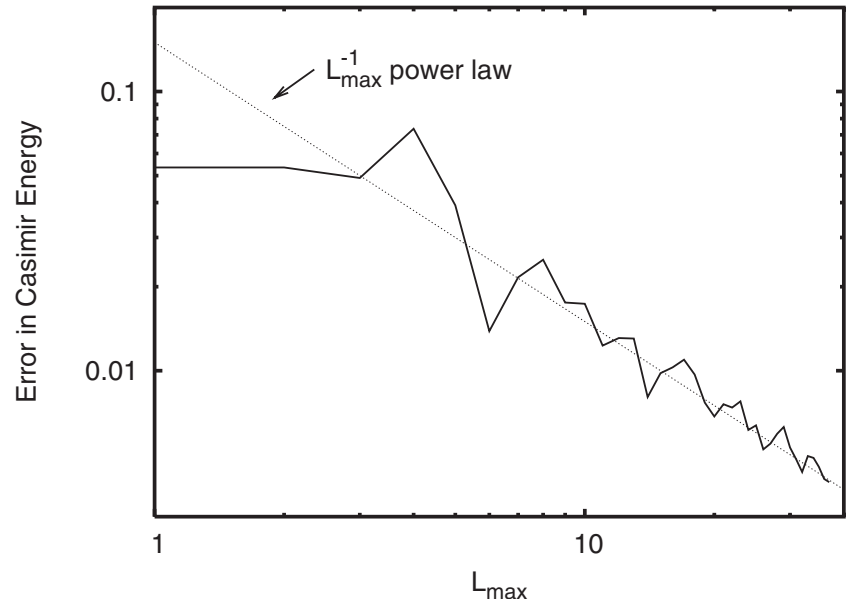

FIG. 4. The error $\left|E_{c}^{L_{\max }}-E_{c}\right|$ in the periodic orbit expansion for the vacuum energy is shown for a star graph with four bonds of length 1.1, 1.6176, 1.2985, and 1.1159, and a Neumann piston at the end of each bond.

$$
E_{c}^{\text {shortest }}=\frac{\pi}{48}\left(1-\frac{24 \ln 2}{\pi^{2} B}+\cdots\right) \sum_{j=1}^{B} \frac{1}{a_{j}} .
$$

This approximation compares well to the analytic result $\frac{\pi}{48}\left(1-\frac{3}{B}\right) \frac{B}{a}$ for $B$ equal-length bonds [Eq. (14)].

The results are illustrated in Fig. 3, where the exact force on each piston in a star graph having either all Dirichlet or all Neumann pistons is compared with the contribution to the force from the shortest periodic orbit. The repulsive behavior in the Neumann case, as well as the attractive behavior in the Dirichlet case, are well explained by considering only the shortest periodic orbit, i.e., the bounce between the piston and the central vertex.

To obtain a better approximation, we may systematically include contributions from longer orbits. In Fig. 4, we show the convergence of the sum (18) when all orbits, including primitive orbits and repetitions, of total length $r L_{p} \leqslant L_{\max }$ are included in the summation. In this example, a star graph with $B=4$ bonds, all Neumann pistons, and unequal bond lengths is used, so the exact answer is obtained to the necessary accuracy from a numerical spectrum as described in Sec. IV. We note that the rate of convergence is given by

$$
\left|E_{c}^{L_{\max }}-E_{c}\right| \sim \frac{1}{L_{\max }},
$$

consistent with the fact that each contribution to Eq. (18) from orbits of length $r L_{p} \in\left[L_{\max }, L_{\max }+\Delta\right]$ scales as $L_{\max }^{-2}$ for large $L_{\max }$, and all such contributions appear preferentially with the same (negative) sign.

In more general situations, involving non-star topologies, more complicated boundary conditions, or nonzero gauge fields, orbits of different length are expected to contribute with random signs to the sum (18). The error made by omitting orbits of length greater than $L_{\max }$ takes the form $\sum_{n=0}^{\infty} D_{n}$, where $D_{n}$, associated with all orbits of total length $r L_{p}$ $\in\left[L_{\max }+n \Delta, L_{\max }+(n+1) \Delta\right]$, scales as $D_{n} \sim\left(L_{\max }+n \Delta\right)^{-2}$, but the $D_{n}$ appear with random (uncorrelated) signs. The

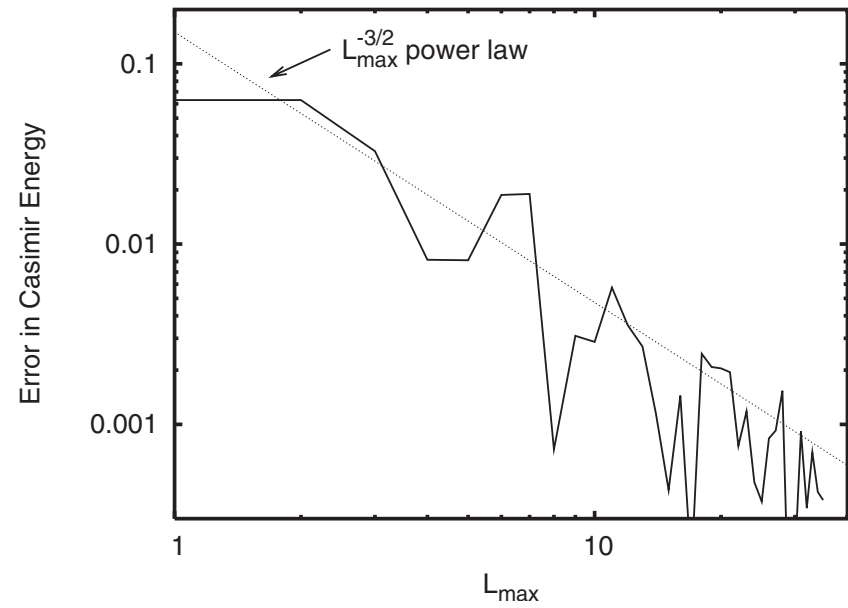

FIG. 5. The error $\left|E_{c}^{L_{\max }}-E_{c}\right|$ is shown for the same quantum graph as in Fig. 4, but with a Dirichlet piston at the end of the first bond.

mean squared error then scales as $\sum_{n=0}^{\infty} D_{n}^{2} \sim \sum_{n=0}^{\infty}\left(L_{\max }\right.$ $+n \Delta)^{-4} \sim L_{\max }^{-3}$, and the root mean square error decays as

$$
\left|E_{c}^{L_{\max }}-E_{c}\right| \sim \frac{1}{L_{\max }^{3 / 2}}
$$

As an example, in Fig. 5, we consider the convergence of the periodic-orbit sum for the same four-bond star graph, but with a Dirichlet instead of Neumann piston on one of the bonds. The behavior is consistent with the faster rate of convergence predicted by Eq. (27).

\section{SUMMARY}

We have seen that unambiguously repulsive as well as unambiguously attractive Casimir forces arise in simple quantum-graph models, and that the sign of the force in a given geometry may often be easily understood in terms of the short periodic orbits of the system. We have also examined (numerically) the rate of convergence of the periodicorbit expansion. Classical-orbit approximations may also be useful for understanding the sign of Casimir forces in higherdimensional piston systems where no analytic solution exists, for example, in two-or three-dimensional chaotic billiards.

\section{ACKNOWLEDGMENTS}

We thank Kimball Milton and the Texas A\&M quantum graph research group (Brian Winn, Gregory Berkolaiko, and Jonathan Harrison) for helpful comments. This research was supported in part by National Science Foundation Grants Nos. PHY-0554849 and PHY-0545390. We thank the Isaac Newton Institute for Mathematical Sciences in Cambridge, U.K., for hosting an extended, supported visit by S.A.F. and a brief visit by J.H.W. 


\section{APPENDIX: INFINITELY PERMEABLE PISTON}

In principle, a repulsive piston can be constructed in the more realistic case of the electromagnetic field in dimension 3 , in analogy with our original one-dimensional model. If the electromagnetic analog of the Dirichlet condition is a perfect conductor, then the analog of the Neumann condition is a material with infinite magnetic permeability, and the Casimir force between slabs of these two different types is repulsive [25]. (A list of references on this topic appears in Ref. [26].) The existence of real materials with sufficient permeability to exhibit Casimir repulsion in the laboratory is controversial [27-29]. Here we merely check that the piston effect discovered by Cavalcanti [3] and the MIT group [4] does not destroy the repulsion shown by less sophisticated calculations. This is not trivial, since the effect arises from the action of the shaft walls on the transverse behavior of the field.

Following Lukosz [1], but in a notation closer to Cavalcanti's (see Fig. 1), we consider a rectangular box with dimensions $a, b_{1}$, and $b_{2}$. As previously exemplified, we can calculate a finite vacuum energy naively, in full confidence that the discarded divergent terms will cancel when a force is calculated for the piston system as a whole. We are interested in the case where the piston (the surface that is free to move) is infinitely permeable but the shaft and the baffle (the rest of the box) are perfect conductors. By the Rayleigh-Dowker argument [30], the energy $\bar{E}_{a}$ of such a box is

$$
\bar{E}_{a}=E_{2 a}-E_{a},
$$

where $E_{a}$ is the energy of a totally conducting box also of length $a$. By differentiation with respect to $a$ (not $2 a$ ), this relation extends to forces and pressures. (Throughout this discussion "pressure" simply means "force per area" without necessarily implying a local pressure independent of position on the wall.) Thus Eq. (11) follows from Eq. (6) by virtue of

$$
-\frac{\pi}{24 a}\left[\frac{1}{2}-1\right]=-\frac{\pi}{24 a}\left[-\frac{1}{2}\right],
$$

and the three-dimensional analogs will involve quantities proportional to

$$
\frac{1}{a^{3}}\left[\frac{1}{8}-1\right]=\frac{1}{a^{3}}\left[-\frac{7}{8}\right] .
$$

When $a \ll b_{j}$, Lukosz calculates an attractive pressure

$$
P_{a}=-\frac{\pi^{2}}{240 a^{4}}
$$

which implies by Eq. (A1) Boyer's formula [25]

$$
\bar{P}_{a}=+\frac{7}{8} \frac{\pi^{2}}{240 a^{4}}
$$

for the box with one permeable wall. The external (long) part of the shaft has length $L-a \gg b_{1}=b_{2}=b$. For this limit, Lukosz finds a repulsive pressure (involving Catalan's constant)

$$
P=+\frac{0.915965}{24 b^{4}}
$$

Just as in Ref. [4], the resulting force is inversely proportional to the cross-sectional area and is independent of $L-a$, so the corresponding energy term is proportional to $L-a$. Therefore, application of Eq. (A1) gives

$$
\bar{P}_{L-a}=P_{L-a}=+\frac{0.915965}{24 b^{4}}
$$

(as ought to be the case, since the nature of the plate at the distant end of the long shaft ought to be irrelevant). To find the total force on the piston, we must reverse the sign of Eq. (A7), add it to Eq. (A5), and multiply by the area $b^{2}$. The point is that the total force is positive if $a \ll b$; the long external part of the shaft has negligible effect in that case.

On the other hand, for a cube Lukosz found that the perfectly conducting box was already repulsive. The formula (A1) does not yield a simple factor $-\frac{1}{2}$ in that case, because the doubled box is no longer a cube. Nevertheless, the graph presented in Ref. [31] shows that $E_{2 a}$ is closer to $\frac{1}{2} E_{a}$ than to $E_{a}$. We conclude that the permeable piston is attractive in the cubical configuration.
[1] W. Lukosz, Physica (Utrecht) 56, 109 (1971).

[2] N. F. Svaiter and B. F. Svaiter, J. Phys. A 25, 979 (1992).

[3] R. M. Cavalcanti, Phys. Rev. D 69, 065015 (2004).

[4] M. P. Hertzberg, R. L. Jaffe, M. Kardar, and A. Scardicchio, Phys. Rev. Lett. 95, 250402 (2005).

[5] G. Barton, Phys. Rev. D 73, 065018 (2006).

[6] M. T. Jaekel and S. Reynaud, J. Phys. I 1, 1395 (1991).

[7] M. Schaden and L. Spruch, Phys. Rev. A 58, 935 (1998).

[8] R. L. Jaffe and A. Scardicchio, Phys. Rev. Lett. 92, 070402 (2004).

[9] M. Schaden, Phys. Rev. A 73, 042102 (2006).

[10] J.-P. Roth, in Théorie du Potentiel, Proceedings of the Colloque Jacques Deny, Lecture Notes in Mathematics, Vol. 1096, edited by G. Mokobodzki and D. Pinchon (Springer, Berlin, 1985), p. 521.
[11] T. Kottos and U. Smilansky, Ann. Phys. (N.Y.) 274, 76 (1999).

[12] P. Kuchment, Waves Random Media 14, S107 (2004).

[13] S. Gnutzmann and U. Smilansky, Adv. Phys. 55, 527 (2006).

[14] P. Kuchment, Waves Random Media 12, R1 (2002).

[15] S. A. Fulling, J. Phys. A 36, 6857 (2003).

[16] J. H. Wilson, Undergraduate Research Fellow thesis, Texas A\&M University, 2007, http://handle.tamu.edu.1969.1/5682

[17] S. A. Fulling, Contemp. Math. 415, 161 (2006).

[18] B. Bellazini and M. Mintchev, J. Phys. A 39, 11101 (2006).

[19] G. Berkolaiko, J. Harrison, and J. H. Wilson (unpublished).

[20] V. Kostrykin and R. Schrader, J. Phys. A 32, 595 (1999).

[21] V. Kostrykin and R. Schrader, Fortschr. Phys. 48, 703 (2000).

[22] V. Kostrykin, J. Potthoff, and R. Schrader, e-print arXiv:math$\mathrm{ph} / 0701009$.

[23] P. Exner and P. Šeba, Rep. Math. Phys. 28, 7 (1989). 
[24] J. D. Bondurant and S. A. Fulling, J. Phys. A 38, 1505 (2005).

[25] T. H. Boyer, Phys. Rev. A 9, 2078 (1974).

[26] D. T. Alves, C. Farina, and E. R. Granhen, Phys. Rev. A 73, 063818 (2006).

[27] D. Iannuzzi and F. Capasso, Phys. Rev. Lett. 91, 029101 (2003).

[28] O. Kenneth, I. Klich, A. Mann, and M. Revzen, Phys. Rev.
Lett. 91, 029102 (2003).

[29] C.-G. Shao, D.-L. Zheng, and J. Luo, Phys. Rev. A 74, 012103 (2006).

[30] J. S. Dowker, Nucl. Phys. B (Proc. Suppl.) 104, 153 (2002).

[31] S. Hacyan, R. Jáuregui, and C. Villarreal, Phys. Rev. A 47, 4204 (1993). 